\title{
SCINTILLATION CAUSTICS IN PLANETARY OCCULTATION LIGHT CURVES
}

\author{
Asantha R. CoOray ${ }^{1}$ and J. L. Elliot ${ }^{2}$ \\ Received 2003 January 17; accepted 2003 March 14; published 2003 March 21
}

\begin{abstract}
We revisit the GSC 5249-01240 light curve obtained during its occultation by Saturn's north polar region. In addition to refractive scintillations, the power spectrum of intensity fluctuations shows an enhancement of power between refractive and diffractive regimes. We identify this excess power as due to high-amplitude spikes in the light curve and suggest that these spikes are due to caustics associated with ray-crossing situations. The flux variation in individual spikes follows the expected caustic behavior including diffraction fringes, which we have observed for the first time in a planetary occultation light curve. The presence of caustics in scintillation light curves requires an inner scale cutoff to the power spectrum of underlying density fluctuations associated with turbulence. Another possibility is the presence of gravity waves in the atmosphere. While occultation light curves previously showed the existence of refractive scintillations, a combination of small projected stellar size and a low relative velocity during the event have allowed us to identify caustics in this occultation. This has led us to reexamine previous data sets, in which we have also found likely examples of caustics.
\end{abstract}

Subject headings: occultations — planets and satellites: individual (Saturn) — solar system: general — turbulence - waves

\section{INTRODUCTION}

The occultation of background stellar sources by foreground solar system objects provides a probe of distant planetary atmospheres (Elliot \& Olkin 1995). The light curves produced during such events allow one to extract information related to the atmospheric thermal structure with spatial resolutions at the distance of the planet of order a few kilometers. In recent years, increasingly sensitive instrumentation has allowed occultation observations with spatial accuracies not limited by instrumental effects but rather by physical parameters associated with the occultation geometry-such as the distance, the relative velocity, and the projected size of the occulted star at the planet.

In Cooray et al. (1998), we presented results from an occultation by Saturn's north polar region of the star GSC 524901240 on 1995 November 20 near the time of the solar-ring plane crossing of Saturn. This occultation had the slowest relative velocity, $0.59 \mathrm{~km} \mathrm{~s}^{-1}$, of 203 predicted events by Saturn between 1990 and 2000 (Bosh \& McDonald 1992). The small projected diameter of the background star, $\sim 0.1 \mathrm{~km}$, allowed subkilometer spatial resolution.

The light curve of GSC 5249-01240 obtained during the occultation at NASA's Infrared Telescope Facility in Mauna Kea, Hawaii, showed an excessive number of high-amplitude spikes. In Cooray et al. (1998), we tentatively identified these large amplitude variations, of over $100 \%$ in some occasions, as due to diffractive scintillations. This suggestion was based on the fact that the projected size of the star at the distance of Saturn, $\sim 0.1 \mathrm{~km}$, was smaller than the Fresnel scale associated with these observations, $\sim 0.7 \mathrm{~km}$. As the light curve probes deeper into the atmosphere, scintillation fluctuation observations are well described through a combination of weak and strong refractive scintillation theories (e.g., Narayan \& Hubbard 1988). In the present occultation, owing to the smaller size of the star when compared to the Fresnel scale, we entered a

\footnotetext{
${ }^{1}$ Theoretical Astrophysics, California Institute of Technology, Mail Stop 130-33, Pasadena, CA 91125.

${ }^{2}$ Department of Earth, Atmospheric, and Planetary Sciences and Department of Physics, Massachusetts Institute of Technology, Cambridge, MA 02139.
}

regime for the first time where potentially new signatures may be observed.

We recommend the reader to Cooray et al. (1998) for observational details. There we also present an analysis based on the temperature structure of Saturn's north polar region. Here we study the light curve under the context of scintillation theories based on both diffraction and refraction. We show that the light curve contains scintillation caustics involving multiply imaged events; the presence of caustics in our light curve agrees with theoretical predictions made previously by Goodman et al. (1987) with regard to caustics in pulsar light curves. Similarly, while not recognized as such, the same caustics events have also been predicted by French \& Lovelace (1983) owing to ray-crossing situations associated with density fluctuations generated by gravity waves. Our light curve probably provides the best evidence for caustics—whatever their cause.

\section{SCINTILLATION CAUSTICS}

The application of scintillations to stellar occultation light curves, due to either turbulence or coherent gravity waves, has been widely discussed in the literature (e.g., Young 1976; French \& Lovelace 1983; Narayan \& Hubbard 1988). In general, scintillation processes are expected from fluctuations in the refractivity, in the case of stellar occultations, with a powerlaw spectrum of the form

$$
\begin{gathered}
Q(\boldsymbol{k})=Q_{0}\left(k^{2}+k_{\text {out }}^{2}\right)^{-\beta / 2} \exp \left(-\frac{k^{2}}{2 k_{c}^{2}}\right) \approx k^{-\beta}, \\
k_{\text {out }}<k<k_{c},
\end{gathered}
$$

where $k$ is the spatial wavenumber and $k_{\text {out }}$ and $k_{c}$ are outer and inner cutoff scales of the fluctuation power spectrum. In the case of an exponential atmosphere, the outer cutoff scale is related to the atmospheric scale height, $H$ (French \& Lovelace 1983).

Between the two cutoff scales, the usual assumption of a power law in density fluctuations leads to two separate and well-known regimes of scintillations involving refraction and diffraction. If the power density spectrum has an inner scale 


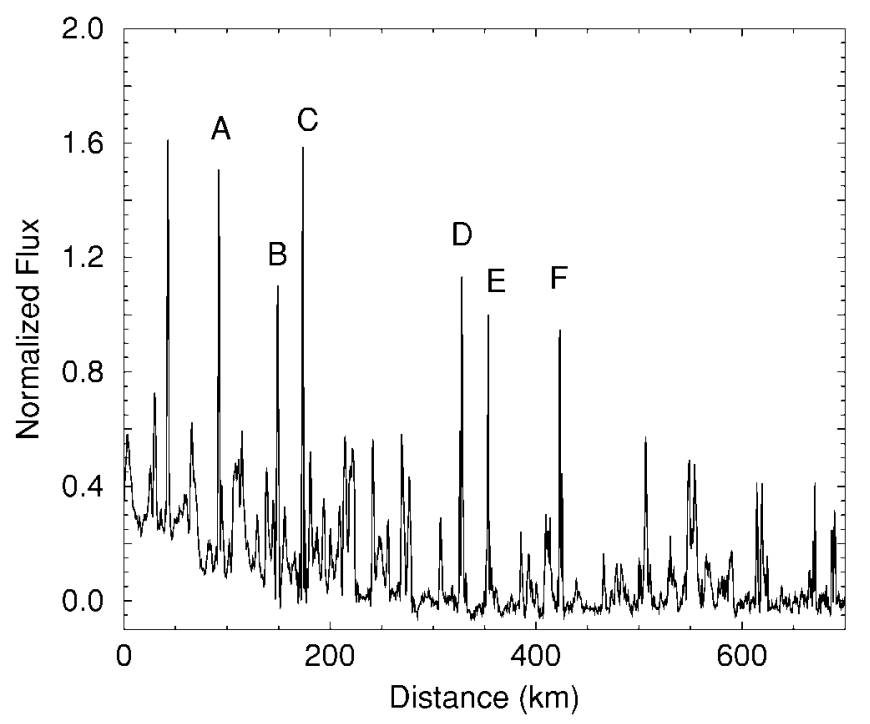

Fig. 1.-GSC 5249-01240 light curve during the occultation by Saturn's north polar region and discussed in Cooray et al. (1998). The existence of high-amplitude short-duration spikes are clearly visible. Note that these appear as pairs, which is an indication for caustics. The labeled spikes are shown in Fig. 3.

cutoff between the refractive and diffractive regimes, Goodman et al. (1987) showed that there is excess power in an intermediate regime due to caustic events and the power spectrum of intensity fluctuations, $P(k)$, scales as $k^{-2}$. In the case of stellar occultations, these caustics can be associated with ray-crossing situations, due to either turbulence or gravity waves (French $\&$ Lovelace 1983). The flux power spectrum in the presence of caustics can be broken to three regimes-refractive, intermediate, and diffractive-with

$$
\begin{gathered}
k^{2} P(k)= \\
\begin{cases}Q_{0} r_{\mathrm{F}}^{\alpha}\left(k r_{\mathrm{F}}\right)^{4-\alpha} \exp \left[-\frac{1}{2}\left(\frac{k}{k_{\text {ref }}}\right)^{2}\right], & k<k_{\text {cref }}, \\
\frac{\pi}{\sqrt{2} \alpha(2-\alpha)|A|}\left(r_{c} / r_{\mathrm{F}}\right)^{4-\alpha}, & k_{\text {cref }}<k<k_{\text {cdif }}, \\
\frac{\pi}{\alpha|A|}\left(r_{c} / r_{\mathrm{F}}\right)^{2-\alpha}\left(k r_{\mathrm{F}}\right)^{2} \exp \left[-\frac{1}{2}\left(\frac{k}{k_{\mathrm{dif}}}\right)^{2}\right], & k>k_{\text {cdif }},\end{cases}
\end{gathered}
$$

respectively, where $\alpha=\beta-2$. Following Goodman \& Narayan (1985), we write

$$
A=\frac{\Gamma[(4-\alpha) / 2]}{2^{\alpha} \pi \alpha(\alpha-2) \Gamma[(\alpha+2) / 2]} Q_{0} r_{\mathrm{F}}^{\alpha}
$$

The various length scales involved in equation (2) are (1) the Fresnel scale, $r_{\mathrm{F}}=(\lambda D / 2 \pi)^{1 / 2}$, when wavelength of observations is $\lambda$ and distance to the scattering screen is $D$; (2) the refractive scale, $r_{\text {ref }}=\theta D$, when the scatter-broadened image size is $\theta$ (eq. [2.10] of Narayan \& Hubbard 1988); (3) the diffractive scale, $r_{\mathrm{dif}}=r_{\mathrm{F}}^{2} / r_{\mathrm{ref}}$, that determines the fringe separation; (4) the inner cutoff scale of density fluctuations, $r_{c}=2 \pi / k_{c}$; (5) the cutoff scale of refractive scintillations, $r_{\text {cref }}=r_{\text {ref }}$ if $\beta<4$ or $r_{\text {cref }}=r_{\text {ref }}\left(r_{c} / r_{\text {ref }}\right)^{(\beta-4) / 2}$ if $\beta>4$; and, finally, (6) the cutoff scale associated with diffractive scintillations, $r_{\text {cdif }}=r_{\mathrm{F}}^{2} / r_{c}$. These
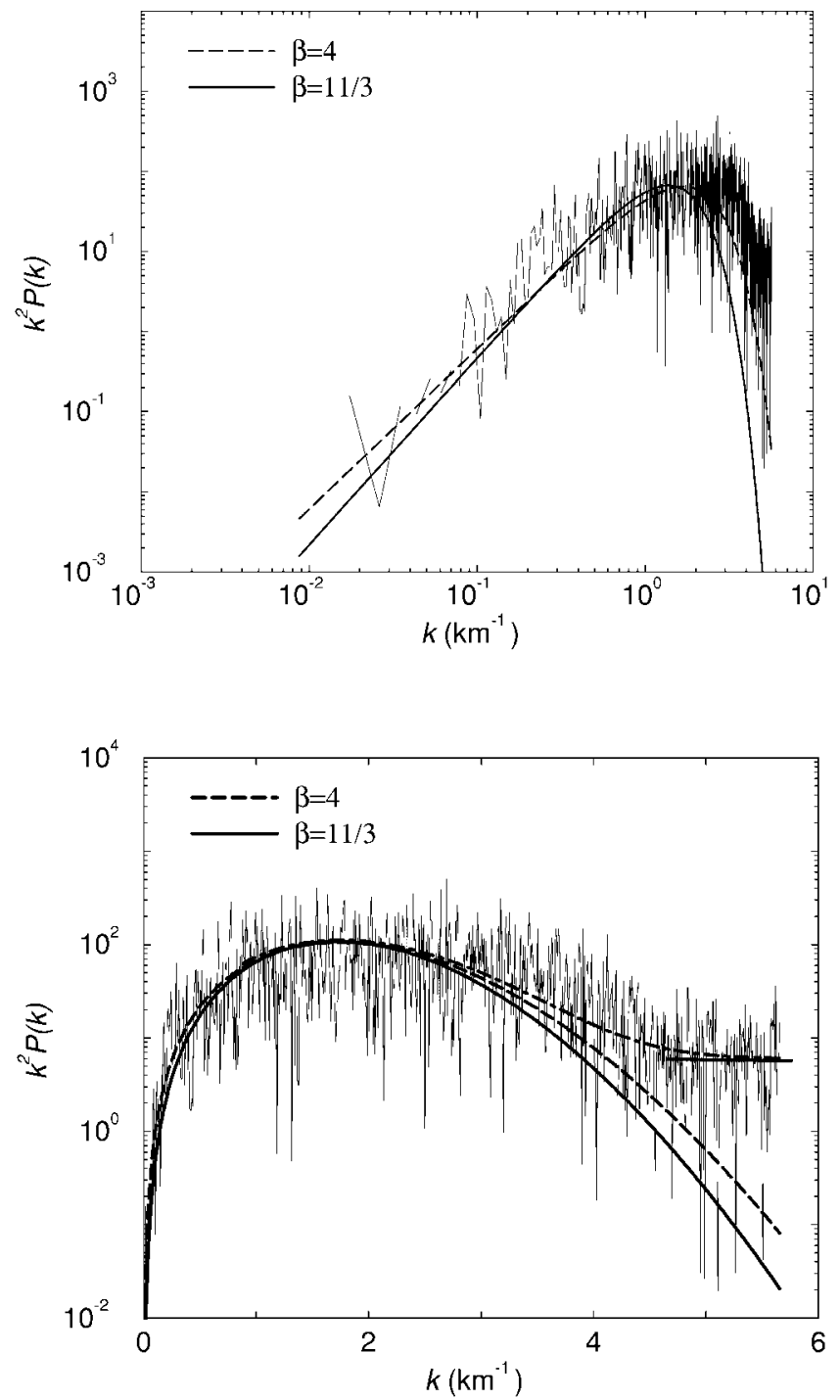

FIG. 2.-Power spectrum of intensity fluctuations. The solid and dashed curves are based on fits to the power spectrum with wavenumbers less than $3 \mathrm{~km}^{-1}$ and using eq. (2) with assumed power laws for the underlying density field fluctuation power spectrum. The peak, at wavenumbers near $1 \mathrm{~km}^{-1}$, is associated with refractive scintillations, while an additional peak, which we have not probed, is expected at high wavenumbers owing to diffractive scintillations. In the bottom panel, we concentrate on the high-wavenumber region where, to the right, we see an indication for the flattening of power at wavenumbers above $4 \mathrm{~km}^{-1}$. Such a flattening is expected when caustics are present in the light curve in addition to simple refraction (Goodman et al. 1987).

length scales are interrelated such that $r_{\text {ref }} r_{\text {dif }}=r_{c} r_{\text {cdif }}=r_{\mathrm{F}}^{2}$. Here length scales, $r$, are related to wavenumbers via $k=2 \pi / r$. In the case of stellar occultations, note that the Fresnel scale decreases as one probes more deeply into the atmosphere.

In Figure 1, we show the light curve of GSC 5249-01240 during the occultation by Saturn. The existence of highamplitude spikes are clearly visible. The relevant parameters are relative velocity, $v_{\perp}=0.586 \mathrm{~km} \mathrm{~s}^{-1}$; distance to Saturn, $D=$ $9.194 \mathrm{AU}$; wavelength, $\lambda=2.28 \mu \mathrm{m}$; and integration time, $t_{\text {int }}=0.947 \mathrm{~s}$. The Fresnel scale is $\sim 0.71 \mathrm{~km}$, while the resolution of the light curve is $\sim 0.55 \mathrm{~km}$. Based on colors, the projected radius of the star at the distance of Saturn is $\sim 0.1 \mathrm{~km}$. For the rest of the discussion, we renormalize the light curve based on model fits (Cooray et al. 1998) and remove the mean flux and only consider fluctuations with respect to this mean.

The power spectrum of intensity fluctuations, $P(k)$, is presented in Figure 2, where we plot $k^{2} P(k)$, which represents 


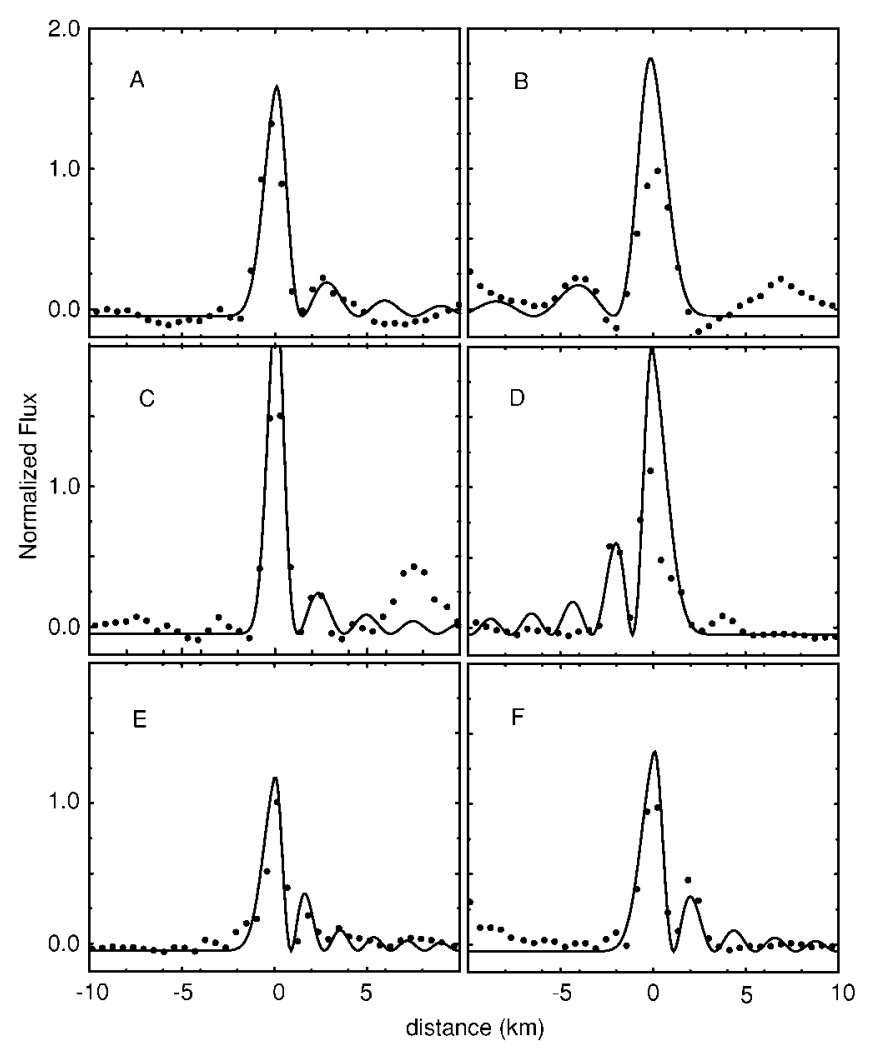

FIG. 3.-Individual spikes as labeled in Fig. 1. The model fits, shown as solid lines, are based on caustic descriptions with an oscillation due to diffraction. The pairs A-B and C-D show the expected right- and left-handed one-sided decrease in flux associated with caustics.

equal weighting in logarithmic wavenumber intervals. In general, this power spectrum is expected to show two distinct peaks associated with refractive and diffractive scintillations (see, e.g., Fig. 3 of Goodman et al. 1987). The data clearly show the existence of the first peak associated with refraction. In the presence of an inner scale cutoff to the underlying density field fluctuations, we expect excess power beyond the refractive peak and a flattening of power at large wavenumbers. We see evidence for such an increase in power and flattening as shown in the bottom plot of Figure 2. Here we have focused on only the large-wavenumber regime that is of interest.

The two solid and dashed curves show fits to the power spectrum under the assumption of $\beta=11 / 3$, a Kolmogorov-type spectrum, and $\beta=4$ in equation (2). With the slope fixed, the model fitting involves two unknown free parameters related to $Q_{0}$ and $k_{\text {ref }}$. In order not to bias model fitting with respect to the excess, we make use of the power spectrum only out to wavenumbers less than $3 \mathrm{~km}^{-1}$. While we can obtain a fit to the whole power spectrum, we cannot explain the apparent flattening of power beyond the turnover at wavenumbers greater than $\sim 4.5 \mathrm{~km}^{-1}$. Note that the flattening cannot be ascribed to simply noise in the power spectrum; white-noise fluctuations will lead to scale-independent power with $P(k) \sim$ constant such that in a $k^{2} P(k)$ plot, one would see a sharp rise.

Using fits to the power spectrum based on theoretical expectations, following equation (2), we determined that for a $\beta=11 / 3$ power law, $r_{\text {ref }}=r_{\text {cref }}=5.7 \pm 1.2 \mathrm{~km}$, and $Q_{0}=$ $380 \pm 130$, at the $68 \%$ confidence level. For a $\beta=4$ power law, these numbers are $5.2 \pm 1.1 \mathrm{~km}$ and $370 \pm 140$, respectively. The errors in fitted parameters are estimated by allowing the normalized light curve to vary randomly up to $\pm 5 \%$; this accounts for, rather conservatively, relative photometric errors and any potential uncertainties associated with the light curve. The associated diffraction scale is $r_{\mathrm{dif}} \sim 0.09 \mathrm{~km}$ or $k_{\mathrm{dif}} \sim$ $70 \mathrm{~km}^{-1}$; we do not probe such small length scales, suggesting that we have not observed regular diffractive scintillations in the present data.

We can determine the inner cutoff scale by comparing the amplitude of flattening to that of the refractive peak in the power spectrum. For the $\beta=11 / 3$ and 4 power laws, we determine this cutoff to be at $r_{c} \sim 4.0 \pm 1.5$ and $4.4 \pm 1.6 \mathrm{~km}$, respectively. The caustic regime extends from the cutoff scale associated with refraction, $r_{\text {cref }} \sim 6 \mathrm{~km}$, to the cutoff scale associated with diffraction, $r_{\text {cdif }} \sim 0.12 \mathrm{~km}$. Following Goodman et al. (1987), the expected flux variations associated with caustic events are given by $(\Delta F / F)^{2} \sim 2\left(r_{c} / r_{\text {ref }}\right)^{2} \ln \left(r_{c} r_{\text {ref }} / r_{\mathrm{F}}^{2}\right)$. Based on derived values, we find $(\Delta F / F) \sim 1.9$, which is consistent although somewhat higher than the observed amplitudes of spikes in the light curve.

The presence of caustics can also be verified by inspecting the intensity profile of individual events since individual caustics are expected to show the $1 / \sqrt{d}$ behavior in the flux variation with distance, $d$, as the source moves away from the critical line. In the case of stellar occultation light curves, such a distance dependence is expected in the observer plane such that the intensity varies as $I(d)=I_{0}+\Theta(d) a_{0} d^{-1 / 2}$, where $\Theta(d)$ is the step function, $a_{0}$ is the caustic strength, and $I_{0} \sim 0$ is the background flux. This intensity dependence, at Saturn, can be written using the fact that the mapping between the source and observer plane involves $d=r_{\text {cref }}\left(x / r_{c}\right)^{2}$ and $a_{0} \sim r_{c}^{2} / r_{\text {cref }}^{3 / 2}$ (Goodman et al. 1987), where $x$ is measured distance in the scatterer plane. This leads to an intensity decrease of $I(x) \sim A_{0} / x$ with $A_{0} \sim r_{c}^{3} / r_{\text {cref }}^{2} \sim 1.9$. In addition to this one-sided drop in flux, caustics are also expected to show the presence of diffraction with an oscillatory pattern superposed on the intensity variation.

In Figure 3, we summarize our model descriptions of individual high-amplitude spikes. While the expected width of the caustic is a priori known, $\sim\left(r_{\mathrm{ref}} r_{\mathrm{F}}^{4} / r_{c}^{2}\right)^{1 / 3}$ (Goodman et al. 1987), we have allowed for smoothing with a Gaussian profile, $\exp \left(-x^{2} / 2 \sigma^{2}\right)$, and treated the width as an unknown parameter. Individual caustic curve model fits to spikes are determined by three parameters: the caustic strength $A_{0}$, the smoothing width, and the oscillation spacing due to diffraction. From fits to six spikes, we determine $A_{0}=1.8 \pm 0.4$, consistent with the expectation value based on parameters derived from the power spectrum analysis. The smoothing width, $\sigma$, is determined to be of order $0.5 \pm 0.2 \mathrm{~km}$ and is consistent with the expected width of order $0.45 \mathrm{~km}$. Note that the caustic width is higher than the size of the star, $\sim 0.1 \mathrm{~km}$, based on its spectrum. While Goodman et al. (1987) considered the case of a background point source, in our case with the finite size for the star, the final caustic width is likely to be smoothed by the projected star size at the distance of Saturn.

Note that caustic spikes appear in pairs in the light curve; such a pairing may lead one to conclude that the occultation, in fact, involves a binary instead of a single star similar to the $\beta$ Sco occultation by Jupiter (Elliot, Rages, \& Veverka 1976). There is one observation that rules out this possibility; in our spikes, we see the alternating right- and left-handed behavior associated with the one-sided decrease in flux. This is naturally associated with caustics.

Before discussing our main results, we note that previous studies on refractive scintillations in occultation light curves have shown evidence for anisotropic inhomogeneities in underlying fluctuations such that in equation (1), $k^{2}=\rho^{2} k_{x}^{2}+$ $k_{y}^{2}$ with $\rho>1$ (Narayan \& Hubbard 1988). In the presence of significant anisotropy, $\rho \gg 1$, the cutoff we have observed is 
simply related to the dominant dimension, and what we have observed through caustics is likely to be the finite size of inhomogeneities in Saturn's atmosphere in the vertical direction. While for the rest of the discussion we ignore the presence of an anisotropic medium, we plan to address this issue as part of a detailed analysis of several light curves of the same occultation event obtained with the Hubble Space Telescope.

\section{DISCUSSION}

Among the two processes for strong scintillation, refraction has been previously studied. The second, diffractive scintillations, was not expected to contribute given the required high resolution to see its effects and the small stellar size required not to average out the diffractive pattern (e.g., Narayan \& Hubbard 1988). In the case of the occultation of GSC 5249-01240 by Saturn, the projected size of the star was smaller than the Fresnel scale and the relative velocity during the event was of order $0.5 \mathrm{~km} \mathrm{~s}^{-1}$. This, in combination, allowed subkilometer spatial resolution.

The light curve contains distinct signatures of sharp and highamplitude spikes; such a high number of them has not been observed previously. While we explored the regime below refractive scintillations, the presence of diffractive scintillations require spatial resolutions less than $0.1 \mathrm{~km}$, below what we have probed. The diffractive scintillations are expected to produce flux variations of order $100 \%$ at regular intervals. In our case, we see high-amplitude spikes at irregular intervals. The spikes, however, can be described through caustics whose presence requires an inner cutoff to fluctuations in the underlying density power spectrum (Goodman et al. 1987). Such a cutoff may also be responsible for the ray crossing one needs for the formation of caustics (French \& Lovelace 1983). Following the notation of French \& Lovelace (1983), the present light curve has an intensity fluctuation strength, $A$, of 14 and a normalized star size of $d_{*}=0.05 r_{\mathrm{F}}$. The strength of intensity fluctuations suggests fractional fluctuations in the refractivity, $\Delta \nu / \bar{\nu}$, of order $4 \times$ $10^{-3}$. If underlying fluctuations are due to gravitational waves, under geometrical arguments, ray crossing is expected when vertical wavelengths are less than $2 \pi H /(\Delta \nu / \bar{\nu})^{-2 / 3} \sim H / 6$ or $7 \mathrm{~km}$ since $H \sim 40 \mathrm{~km}$ (Cooray et al. 1998). This value is the constraint when only a single wave is present in the atmosphere. The case with a spectrum of waves is analogous to that described by Goodman et al. (1987).

The presence of caustics is aided by several observations that involve the detection of excess power in the power spectrum of intensity fluctuations and the flattening of the same power spectrum in the intermediate regime between refractive and diffractive scintillations. The intensity variation in individual spikes is also well described through the caustic description with a modulation due to diffraction. The parameters from the power spectrum are consistent with model descriptions to the spikes aiding the suggestion for the presence of caustics. While we have not investigated in detail, we may have found occasional examples of caustic spikes in previous occultation light curves, such as the "camel" spike of Elliot \& Veverka (1976). These, however, do not contain the signature of diffraction fringes as observed in the present occultation.

From our data, we determined the inner scale cutoff of the underlying density fluctuation power spectrum to be around $4.5 \mathrm{~km}$. The density fluctuations are generally considered to be caused by processes involving either turbulence or gravity waves (e.g., Elliot \& Veverka 1976; Young 1976; French \& Lovelace 1983; Narayan \& Hubbard 1988). It is likely that the cutoff may be associated with viscous dissipation of wave energy. In Cooray et al. (1998), we found an increase in temperature of $\sim 15 \mathrm{~K}$ between $\sim 10$ and $1 \mu$ bar of the region probed by this occultation. Using our cutoff scale and parameters appropriate for this occultation, we extend the calculation by Roques et al. (1994) on heating by wave decay to estimate an energy transfer rate of $\sim 10^{-7} \mathrm{ergs} \mathrm{cm}^{-3} \mathrm{~s}^{-1}$.

The detection of caustics and, for the first time, diffraction fringes in an occultation light curve suggest that we entered a regime that was previously unexplored. We believe that it was a combination of the small velocity, high sampling rate, and small projected size of the occulted star that allowed us to detect caustics clearly. When observing potential occultations, we suggest events with such favorable conditions be given additional priority.

We thank R. Blandford, P. Goldreich, D. Holz, L. Koopmans, and R. Sari for useful discussions. We acknowledge support from the Department of Energy DE-FG03-92-ER40701 and the Sherman Fairchild Foundation (at Caltech) and NASA grant NAG5-10444 and NSF grant AST-0073447 (at MIT).

\section{REFERENCES}

Bosh, A. S., \& McDonald, S. W. 1992, AJ, 103, 983

Cooray, A. R., Elliot, J. L., Bosh, A. S., Young, L. A., \& Shure, M. A. 1998, Icarus, 132, 298

Elliot, J. L., \& Olkin, C. B. 1995, Annu. Rev. Earth Planet. Sci., 24, 89

Elliot, J. L., Rages, K., \& Veverka, J. 1976, ApJ, 207, 994

Elliot, J. L., \& Veverka, J. 1976, Icarus, 27, 359

French, R. G., \& Lovelace, R. V. E. 1983, Icarus, 56, 122
Goodman, J., \& Narayan, R. 1985, MNRAS, 214, 519

Goodman, J. J., Romani, R. W., Blandford, R. D., \& Narayan, R. 1987, MNRAS, 229, 73

Narayan, R., \& Hubbard, W. B. 1988, ApJ, 325, 503

Roques, F. B., et al. 1994, A\&A, 288, 985

Young, A. T. 1976, Icarus, 27, 335 